$\mathbb{N} \mathbf{K I T}$

Karlsruhe Institute of Technology

\title{
He-cooled Divertor: Study on Low-Temperature Design Using Ta Alloy as Thimble Material
}

\author{
${ }^{1}$ P. Norajitra, ${ }^{1}$ W. Basuki, ${ }^{2}$ Bostjan Koncar, ${ }^{1}$ L. Spatafora \\ ${ }^{1}$ Karlsruhe Institute of Technology (KIT), Institute for Applied Materials (IAM) P.O. Box 3640, 76021 Karlsruhe, Germany \\ 2Jožef Stefan Institute, Jamova 39, 1000 Ljubljana, Slovenia
}

- Reference design HEMJ [1] (Fig. 1)

- Nominal heat flux $10 \mathrm{MW} / \mathrm{m}^{2}$.

- Cooling: impinging multiple helium jets (10 MPa, $\left.600^{\circ} \mathrm{C}\right)$. - Hex. W tile (18mm A/F) brazed to WL10 thimble ( 15 $x 1 \mathrm{~mm})$, joined with ODS Eurofer back bone structure.

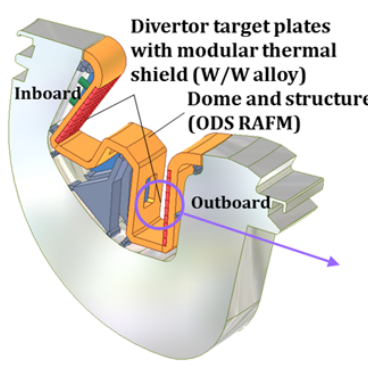

Divertor cassette
9-Finger module

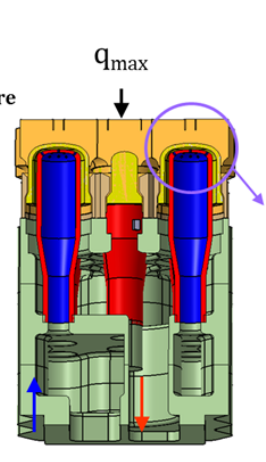

1-Finger module

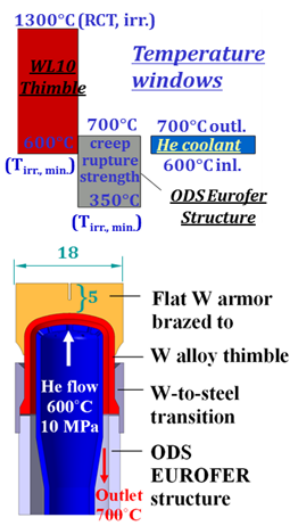

Fig. 1: Reference HEMJ design: Heat removal by helium jet impingement (10 $\mathrm{MPa}, 600^{\circ}$

- Problem: unknown irradiated data for $\mathbf{W}$ materials

\begin{tabular}{|l|l|c|c|}
\hline Material & \multicolumn{1}{|c|}{$\begin{array}{c}\text { DBTT unirr. } \\
\left({ }^{\circ} \mathrm{C}\right)\end{array}$} & $\begin{array}{c}\Delta \mathrm{DBTT} @ \mathrm{dpa} / \mathrm{T}_{\text {irr. }}\left({ }^{\circ} \mathrm{C}\right) \\
\text { Eurofer [3] }\end{array}$ & $\begin{array}{c}\mathrm{T}_{\text {irr., }} \Delta \mathrm{DBTT}, \min \text { * * } \\
\left({ }^{\circ} \mathrm{C}\right)\end{array}$ \\
\hline $\mathrm{W}[4]$ & $\begin{array}{l}\sim 650 \\
(\mathrm{rod}, \varnothing 20 \mathrm{~mm})\end{array}$ & unknown & 350 \\
\hline WL10 [4] & $\begin{array}{l}400-450 \\
(\mathrm{rod}, \varnothing 20 \mathrm{~mm})\end{array}$ & unknown & unknown \\
\hline T-111 [2] & -190 & unknown & unknown \\
\hline
\end{tabular}

*above which only minor DBTT shift is expected

- Alternative HEMJ design with T-111 thimble (Fig. 2)
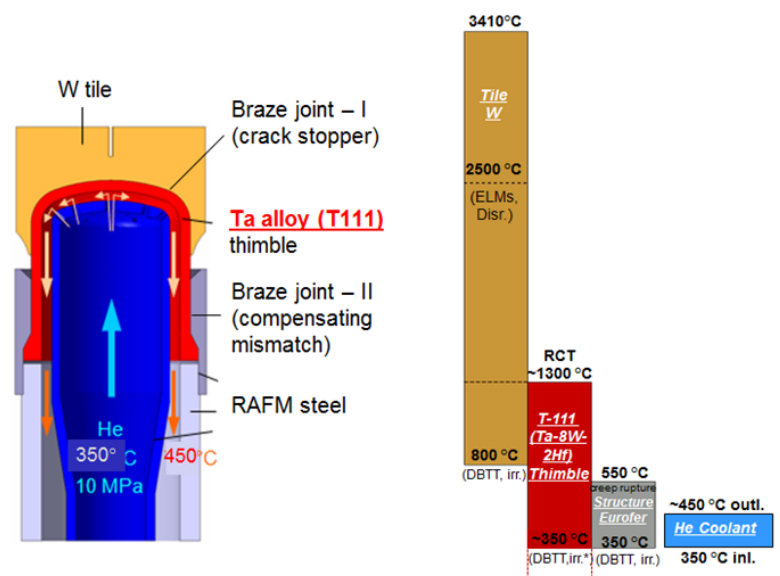

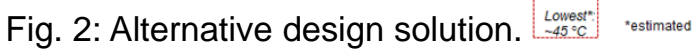

[1] P. Norajitra et al., Fusion Eng. Des. 83 (2008) 893-902.

[2] NASA Technical Note, NASA TN D-5873, Survey of Properties of T-111.

[3] E. Gaganidze et al, J. Nucl. Mater. 355 (2006) 83-88.

[4] M. Rieth, http://bibliothek.fzk.de/zb/veroeff/79094.pdf,

http://bibliothek.fzk.de/zb/veroeff/81120.pdf, retrieved 03.06.2013.
- Advantages of T-111 [2] as alternative thimble material - High creep resistant at $\mathrm{T}-980-1310^{\circ} \mathrm{C}$.

a Extremely low DBTT of $-196^{\circ} \mathrm{C}$.

- Good formability and weldability.

- Corrosion resistance to liquid alkali metals ( $\mathrm{Li}, \mathrm{Na}, \mathrm{K}$ ).

- Design Verification by CFD and FEM Analyses

Table 1: Boundary conditions (top) and result summary (bottom) of CFD analysis.

\begin{tabular}{|l|c|c|}
\hline & $\begin{array}{c}\text { Reference case } \\
\text { (Thimble WL10) }\end{array}$ & Thimble T-111 \\
\hline $\begin{array}{l}\text { Mass flow rate per finger } \\
(\mathrm{g} / \mathrm{s})\end{array}$ & 6.8 & 6.8 \\
\hline He inlet temperature $\left({ }^{\circ} \mathrm{C}\right)$ & 634 & 350 \\
\hline He pressure $(\mathrm{MPa})$ & 10 & 10 \\
\hline He density $\left(\mathrm{kg} / \mathrm{m}^{3}\right)$ & 5.2 & 7.0 \\
\hline Heat flux $\left(\mathrm{MW} / \mathrm{m}^{2}\right)$ & 10 & 10 \\
\hline $\begin{array}{l}\text { Volumetric heat } \\
\text { generation }\left(\mathrm{MW} / \mathrm{m}^{3}\right)\end{array}$ & 17 & 17 \\
\hline
\end{tabular}

\begin{tabular}{|l|c|c|}
\hline & $\begin{array}{c}\text { Reference case } \\
\text { (Thimble WL10) }\end{array}$ & Thimble T-111 \\
\hline Max. tile temp. $\left({ }^{\circ} \mathrm{C}\right)$ & 1783 & 1606 \\
\hline Max. thimble temp. $\left({ }^{\circ} \mathrm{C}\right)$ & 1201 & 1055 \\
\hline He outlet temp. $\left({ }^{\circ} \mathrm{C}\right)$ & 712 & 429 \\
\hline Max. He velocity $(\mathrm{m} / \mathrm{s})$ & 245 & $\sim 170$ \\
\hline Pressure loss $(\mathrm{MPa})$ & 0.12 & 0.08 \\
\hline
\end{tabular}

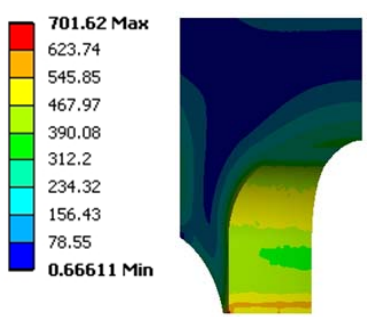

Tile *a rigid joint tile/thimble conservatively assumed.

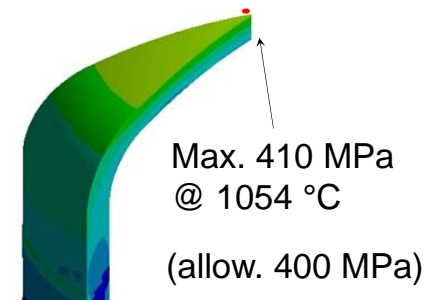

Thimble
Fig. 3: Von Mises equivalent stress* (MPa) by ANSYS for T-111 case, (heat flux $10 \mathrm{MW} / \mathrm{m}^{2}, \mathrm{~T}_{\mathrm{He}} 350^{\circ} \mathrm{C}$ ).

\section{- Conclusion}

- An alternative solution with T-111 material may satisfy the requirements on the ductility of thimble structure.

- The chosen coolant temperature of $350^{\circ} \mathrm{C}$ allows for the simplistic application of Eurofer base material instead of ODS Eurofer. 